\title{
Increased Mortality After Prosthetic Joint Infection in Primary THA
}

\author{
Per Hviid Gundtoft MD, PhD, Alma Becic Pedersen MD, PhD, DMSc, \\ Claus Varnum MD, PhD, Søren Overgaard MD, DMSc
}

Received: 13 September 2016/Accepted: 16 February 2017/Published online: 24 February 2017

(C) The Association of Bone and Joint Surgeons $\mathbb{R} 2017$

\begin{abstract}
Background Revision for prosthetic joint infection (PJI) has a major effect on patients' health but it remains unclear if early PJI after primary THA is associated with a high mortality.

Questions/Purposes (1) Do patients with a revision for PJI within 1 year of primary THA have increased mortality compared with patients who do not undergo revision for any reason within 1 year of primary THA? (2) Do patients who undergo a revision for PJI within 1 year of primary THA have an increased mortality risk compared with
\end{abstract}

The institution of one or more of the authors (SO) has received funding from Eli Lilly (Nybrovej, Denmark); Biomet Inc (Warsaw, IN, USA); DePuy (Beeston, UK); Protesekompagniet (Roskildevej, Denmark); and Zimmer Herstedvang, Denmark).

All ICMJE Conflict of Interest Forms for authors and Clinical Orthopaedics and Related Research ${ }^{\circledR}$ editors and board members are on file with the publication and can be viewed on request.

Each author certifies that his or her institution approved or waived approval for the human protocol for this investigation and that all investigations were conducted in conformity with ethical principles of research.

This work was performed at the Department of Orthopaedic Surgery and Traumatology, Odense University Hospital, Odense, Denmark; and the Department of Orthopaedics, Kolding Hospital, Kolding, Denmark.

P. H. Gundtoft ( $₫)$

Department of Orthopaedics Surgery, Kolding Hospital,

Kolding, Denmark

e-mail: per.hviid.gundtoft@rsyd.dk

P. H. Gundtoft, S. Overgaard

Department of Orthopaedic Surgery and Traumatology, Odense

University Hospital, Odense, Denmark

P. H. Gundtoft, S. Overgaard

Institute of Clinical Research, University of Southern Denmark,

Odense, Denmark patients who undergo an aseptic revision? (3) Are there particular bacteria among patients with PJI that are associated with an increased risk of death?

Methods This population-based cohort study was based on the longitudinally maintained Danish Hip Arthroplasty Register on primary THA performed in Denmark from 2005 to 2014. Data from the Danish Hip Arthroplasty Register were linked to microbiology databases, the National Register of Patients, and the Civil Registration System to obtain data on microbiology, comorbidity, and vital status on all patients. Because reporting to the register is compulsory for all public and private hospitals in Denmark, the completeness of registration is $98 \%$ for primary THA and $92 \%$ for revisions (2016 annual report). The mortality risk for the patients who underwent revision for PJI within 1 year from implantation of primary THA was compared with (1) the mortality risk for patients who did not undergo revision for any reason within 1 year of primary THA; and (2) the mortality risk for patients who underwent an aseptic revision. A total of 68,504 primary THAs in 59,954 patients were identified, of those 445 primary THAs underwent revision for PJI, 1350 primary THAs underwent revision for other causes and the remaining 66,709 primary THAs did not undergo revision.

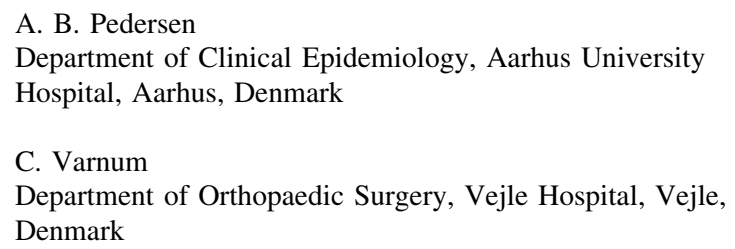


Patients were followed from implantation of primary THA until death or 1 year of followup, or, in case of a revision, 1 year from the date of revision.

Results Within 1 year of primary THA, 8\% (95\% CI, $6 \%-11 \%$ ) of patients who underwent revision for PJI died. The adjusted relative mortality risk for patients with revision for PJI was 2.18 (95\% CI, 1.54-3.08) compared with the patients who did not undergo revision for any cause ( $p$ $<0.001)$. The adjusted relative mortality risk for patients with revisions for PJI compared with patients with aseptic revision was 1.87 (95\% CI, 1.11-3.15; $\mathrm{p}=0.019)$. Patients with enterococci-infected THA had a 3.10 (95\% CI, 1.66$5.81)$ higher mortality risk than patients infected with other bacteria $(\mathrm{p}<0.001)$.

Conclusions Revision for PJI within 1 year after primary THA induces an increased mortality risk during the first year after the revision surgery. This study should incentivize further studies on prevention of PJI and on risk to patients with the perspective to reduce mortality in patients who have had THA in general and for patients with PJI specifically.

Level of Evidence Level III, therapeutic study.

\section{Introduction}

One of the most-feared complications in THA is prosthetic joint infection (PJI) because it is associated with more readmissions [19], longer length of stay [23], and subsequent revisions [5]. Furthermore, one study including THA and TKA showed that there is an increased mortality risk within 1 year after revision for PJI [27]. However, not all studies have identified PJI to be associated with a higher mortality risk [4, 24], and so far studies of PJI and mortality have been based on a mixture of THA and TKA $[24,27]$, patients from a single institution [4, 24, 27], and without a maximum defined interval from primary THA surgery to revision for PJI [4, 24, 27].

Preferably, studies of mortality risk after PJI in THA should be performed on large national or international unselected cohorts. National arthroplasty registers such as the Danish Hip Arthroplasty Register offer such large cohorts, but these national registers are hampered by their accuracy in registration of revision resulting from PJI $[6,8,11]$. However, we have achieved data of very-high validity on PJI from the Danish Hip Arthroplasty Register when data are linked with data from the microbiology databases [7]. Furthermore, the national civil registration system in Denmark allows for complete and precise followup of all Danish citizens.

The objective of this article was to study the mortality risk of patients who were surgically treated for a PJI within
1 year after a primary THA. Specifically, we asked whether (1) patients with a revision for PJI within 1 year after primary THA have an increased 1-year mortality risk compared with patients who did not undergo revision within 1 year after primary THA, (2) patients who undergo revision for PJI have an increased 1-year mortality risk compared with patients who undergo aseptic revision, and (3) there may be particular bacteria among patients with PJI that are associated with an increased risk of death.

\section{Materials and Methods}

We conducted this study in Denmark (population $5,627,235$ in 2014) using longitudinally maintained data from population-based clinical quality and administrative registers. We included patients reported to the Danish Hip Arthroplasty Register with a primary THA from 2005 to 2014. The Danish Hip Arthroplasty Register was linked on a person level with microbiology databases, the National Register of Patients, and the Civil Registration System.

\section{The Danish Hip Arthroplasty Register}

The Danish Hip Arthroplasty Register is a clinical-quality database that contains information on primary THA and revisions performed in Denmark including type of preoperative prophylactic antibiotic treatment before revision. Because reporting to the register is compulsory for all public and private hospitals in Denmark, the completeness of registration is $98 \%$ for primary THA and $92 \%$ for revisions, according to the 2016 annual report [5]. Primary diagnosis and the PJI diagnosis in the register have been validated [7, 15]. The primary THA can be linked to any following revisions by use of the registered operation side and the unique civil registration number. The Danish Hip Arthroplasty Register holds data on various variables including duration of surgery and preoperative prophylactic antibiotics.

\section{The Civil Registration System}

All Danish citizens are assigned a unique and unchangeable civil registration number at date of birth or immigration. The register is updated daily and the prevalence of disappearing persons is only $0.3 \%$, which allows for complete followup for all patients, except those who emigrate from Denmark [20]. The Civil Registration System holds information on date of birth, sex, emigration, and death. The civil registration number is recorded in all Danish registers, which allows for unambiguous linkage. 
The Danish National Register of Patients

This register contains data on discharge diagnosis and date of discharge since 1977. Hospital outpatient and emergency visits have been recorded in the register since 1995. Diagnoses are classified according to the International Classification of Diseases, 8th Revision before 1994 and International Classification of Diseases, 10th Revision since 1994. Data from the Danish National Register of Patients were used to determine the Charlson Comorbidity Index score [16] at the time of implantation of primary THA. Recorded primary and secondary diagnoses were included. All diagnoses used in the Charlson Comorbidity Index score have been validated [21]. Diagnoses in the National Register of Patients are used by the Danish National health and medicine authorities to reimburse departments, which motivates them for reporting to the register.

\section{Microbiology Databases}

All departments of clinical microbiology in Denmark maintain electronic laboratory information systems. Since January 2010 an electronic copy of the microbiology report has automatically been sent on a daily basis from the electronic laboratory information systems to the national Danish Microbiology Database. Data in the register have been validated [2]. Before 2010 information on intraoperative cultures was extracted from the electronic laboratory information system, and after 2010, information was extracted from the national Danish Microbiology Database. The local electronic laboratory information systems and the national Danish Microbiology Database store the information automatically in the databases and the information and coverage should be complete on a national level.

\section{Study Population}

Inclusion criteria were primary THA performed between January 1, 2005, and December 31, 2009, in the Danish provinces of Jutland or Funen ( 3 million inhabitants of a population of 5.7 million in Denmark) or performed in Denmark (5.7 million) between January 1, 2010, and December 31, 2014. The reason why only patients from Jutland and Funen were included before 2010 was that information regarding intraoperative cultures was accessible only from these two providences before 2010. Information regarding intraoperative cultures was extracted manually for patients who before 2010 had a primary THA performed in Jutland or Funen and a subsequent revision performed outside these two provinces. This ensured that all data regarding intraoperative cultures from revisions were included in the study. Exclusion criteria were incorrect civil registration number reported to the Danish Hip Arthroplasty Register, more than one primary THA registered with the same operation side, and missing information regarding operation side or date (Fig. 1). In the case of bilateral THAs, both hips were included. In the first analysis patients were followed until death or 1 year from the primary THA, whichever came first. In the second analysis, patients were followed 1 year from the date of revision. Patients who underwent surgery in Jutland or Funen before 2011 were included in a previous study [6].

Revision was defined as any subsequent operation on the primary THA, including débridement without removal of any part of the prosthesis. A revision was defined as resulting from PJI if either three or more intraoperative cultures showed growth of the same virulent or opportunistic bacteria or "deep infection" was reported to the Danish Hip Arthroplasty Register as the indication for revision. If all five or more intraoperative cultures from the revision were negative, the revision was classified as aseptic, regardless of the registered indication in the Danish Hip Arthroplasty Register. If no intraoperative cultures were taken during surgery the revision was classified as PJI or aseptic based on the reported diagnosis to the Danish Hip Arthroplasty Register alone. This definition was validated in a previous study [7].

We classified the study population in three groups according to the event within 1 year after primary THA (Fig 1): Revision for PJI: all patients with a revision for PJI within 1 year after primary THA; Aseptic revision: all patients with an aseptic revision within 1 year after primary THA; and Nonrevision population: all patients who did not undergo revision for any reason during the first year after primary THA.

A total of 68,504 primary THAs were identified in 59,954 patients. Twenty-eight patients $(0.04 \%)$ with 30 primary THAs emigrated within 1 year of their primary THA. The median age of the patients at the time of the primary THA was 70 years (interquartile range, 62-77 years), and the majority were women (56\%) (Table 1). Within 1 year of followup from the primary THA, 1871 patients had died, accounting for 1907 primary THAs. Of the 68,504 primary THAs, 1795 were revised within 1 year. Four hundred forty-five of these were attributable to a PJI according to our definition (revision for PJI), 1350 underwent aseptic revision, and the remaining 66,709 did not undergo revision for any cause (nonrevision population) (Fig. 1).

\section{Statistics}

The incidence of PJI for the study population was estimated with the cumulative competing risk analysis, 


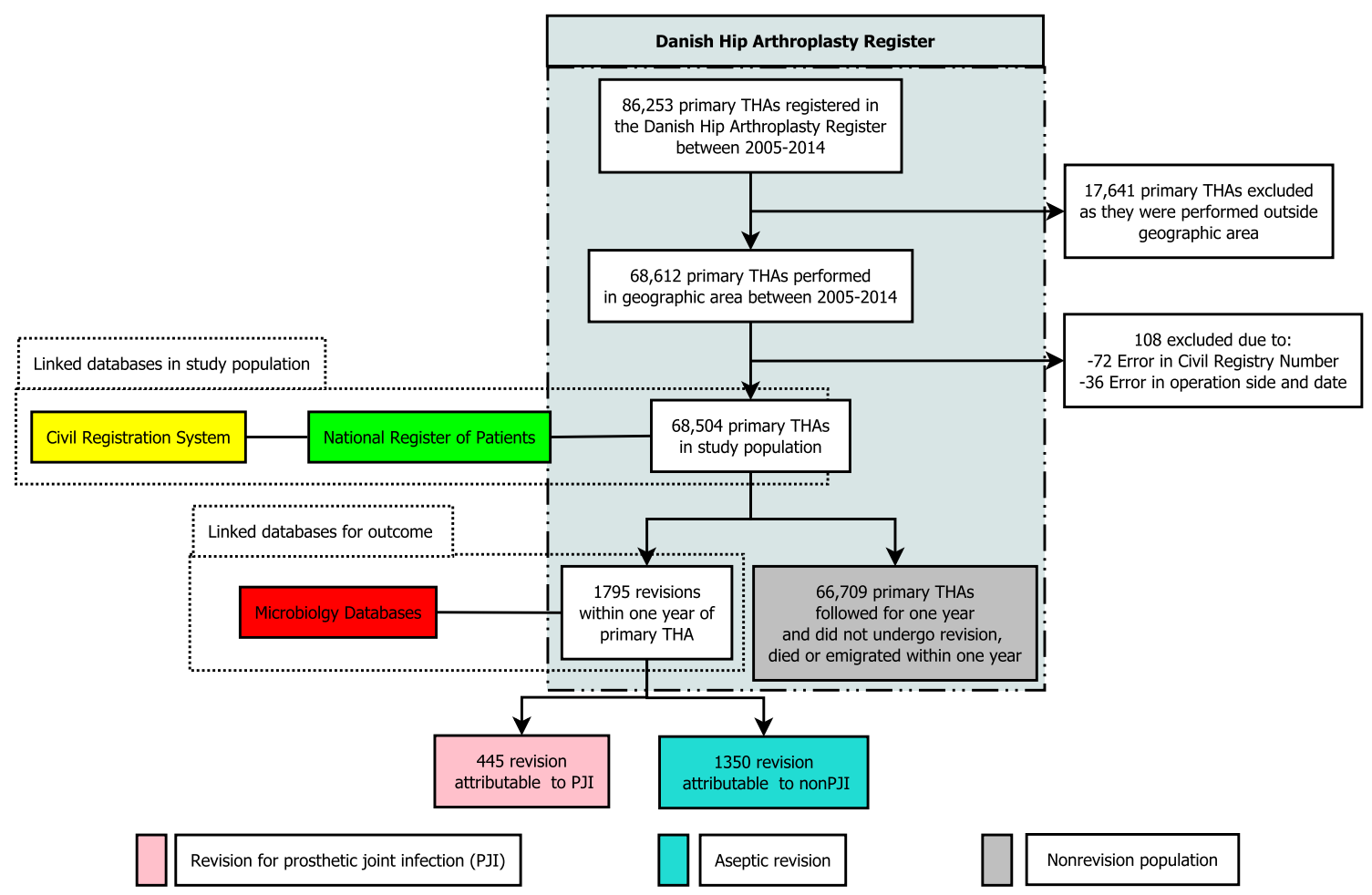

Fig. 1 A flowchart of the study population is shown.

treating death and revisions resulting from other indications than PJI as competing risk. The 1-year incidence of PJI was 0.65 (95\% CI, 0.59-0.71).

The 1-year incidence of mortality was estimated using Kaplan-Meier analysis and for the entire study population was 3\% (95\% CI, 3\%-3\%), but was notably higher if patients underwent revision for PJI (Table 2). We performed three analyses to test our three research questions.

\section{Revision for PJI versus Nonrevision}

We compared the relative mortality risk within 1 year from primary THA for patients who underwent revision for PJI within 1 year of their primary THA with the mortality risk for patients who did not undergo revision for any reason during the first year after primary THA (Fig. 1). All patients were followed until death or 1 year after primary THA. To avoid immortality bias for patients who had revision for PJI, we used the illness-death model in which a time-dependent variable was introduced and the period from the primary THA to PJI revision was classified as nonPJI time [25]. The relative risk was estimated with the pseudovalue method [1]. We adjusted for age $(<60$ years, 60-70, 70-80, $>80$ years), sex, and comorbidity (Charlson Comorbidity Index score: low $=0$, medium $=1-2$, and high $=\geq 3$ ).

\section{Revision for PJI versus Aseptic Revision}

Only patients from the study population who had undergone a revision (PJI or aseptic) were included in this analysis (Fig. 1). The relative mortality risk between patients with a revision for PJI and patients with an aseptic revision was estimated with the pseudovalue method [1]. Patients were followed for a maximum of 1 year from the date of revision surgery, because mortality more than 1 year after revision surgery is less likely to be influenced by PJI. Because the last extraction of data from the Danish Hip Arthroplasty Register was performed June 16, 2016, 16 patients who underwent revisions had a followup shorter than 1 year, but the minimum followup from the date of revision was 8 months.

We adjusted for age, sex, comorbidity, duration of revision surgery (0-60 minutes, 60-90 minutes, 90-120 minutes, $\geq 120$ minutes), and number of secondary revisions.

\section{Revision for PJI: Bacteria Associated With High Mortality}

For revisions for PJI, we further analyzed whether infection with the most-commonly identified bacteria species was associated with higher mortality risk than others 
Table 1. Demographic variables of the three groups

\begin{tabular}{|c|c|c|c|}
\hline Demographic & $\begin{array}{l}\text { Nonrevision population (number of } \\
\text { THAs }=66,709 \text { ) }\end{array}$ & $\begin{array}{l}\text { Aseptic revision (number of } \\
\text { THAs }=1350 \text { ) }\end{array}$ & $\begin{array}{l}\text { Revision for PJI (number of } \\
\text { THAs }=445 \text { ) }\end{array}$ \\
\hline $\begin{array}{l}\text { Age, years: median } \\
\quad \text { (interquartile range) }\end{array}$ & $70(62-77)$ & $70(62-77)$ & $70(64-77)$ \\
\hline Sex: $\%$ female & $56 \%$ & $58 \%$ & $56 \%$ \\
\hline \multicolumn{4}{|l|}{ Comorbidity } \\
\hline Low 0 & $45,629(68 \%)$ & $816(60 \%)$ & $246(55 \%)$ \\
\hline Medium 1-2 & $16,634(25 \%)$ & $399(30 \%)$ & $143(32 \%)$ \\
\hline High $\geq 3$ & $4,446(7 \%)$ & $135(10 \%)$ & $56(13 \%)$ \\
\hline \multicolumn{4}{|l|}{ Primary diagnosis } \\
\hline Osteoarthritis & $56,083(80 \%)$ & $982(73 \%)$ & $312(70 \%)$ \\
\hline $\begin{array}{l}\text { Fracture of either femur or } \\
\text { acetabulum }\end{array}$ & $8138(12 \%)$ & $248(18 \%)$ & $104(23 \%)$ \\
\hline $\begin{array}{l}\text { Avascular necrosis of the } \\
\text { femoral head }\end{array}$ & $1498(2 \%)$ & $59(4 \%)$ & $12(3 \%)$ \\
\hline Arthritis & $825(1 \%)$ & $16(1 \%)$ & $4(1 \%)$ \\
\hline Congenital hip disorder & $2343(4 \%)$ & $33(2 \%)$ & $7(2 \%)$ \\
\hline Other & $822(1 \%)$ & $12(1 \%)$ & $6(1 \%)$ \\
\hline \multicolumn{4}{|l|}{ Number of secondary revisions } \\
\hline 0 & none & $1110(82 \%)$ & $287(65 \%)$ \\
\hline 1 & none & $164(12 \%)$ & $101(23 \%)$ \\
\hline 2 & none & $74(5 \%)$ & $56(13 \%)$ \\
\hline \multicolumn{4}{|l|}{ Duration of revision surgery } \\
\hline 0-60 minutes & none & $322(24 \%)$ & $72(16 \%)$ \\
\hline $60-90$ minutes & none & $436(33 \%)$ & $165(37 \%)$ \\
\hline $90-120$ minutes & none & $296(22 \%)$ & $90(20 \%)$ \\
\hline$\geq 120$ minutes & none & $283(21 \%)$ & $115(26 \%)$ \\
\hline
\end{tabular}

$\mathrm{PJI}=$ prosthetic joint infection.

(Staphylococcus aureus, coagulase-negative staphylococci, enterobacteriaceae, enterococcus, streptococcus, other bacteria groups, and polymicrobial infections). The relative mortality risk was estimated with the pseudovalue method [1].

\section{Results}

\section{Revision for PJI versus Nonrevision Population}

Patients undergoing revision for PJI within 1 year of the index THA were more likely to die during the period of observation than those who underwent revision in the absence of infection. Of those who underwent revision for PJI, 8\% (95\% CI, 6\%-11\%) died within 1 year of the primary THA. In the nonrevision population, which included all patients who did not undergo revision for any cause, 3\% (95\% CI, 3\%-3\%) died, which was notably less than in the revision for PJI group ( $p<0.001)$. The adjusted relative mortality risk for patients with revisions for PJI was 2.18 (95\% CI, 1.54-3.08) compared with the nonrevision population.

\section{Revision for PJI Versus Aseptic Revision}

Patients undergoing revision for PJI were more likely to die within 1 year of the revision surgery than those who underwent aseptic revision, of which 5\% (95\% CI, 4\%$6 \%)$ died $(\mathrm{p}=0.019)$. The adjusted relative mortality risk within 1 year of revision was 1.87 (95\% CI, 1.11-3.15) for revision for PJI compared with aseptic revision.

\section{Bacteriology and Risk of Death}

Patients with PJI caused by enterococci had higher mortality risk within 1 year of revision than if PJI were caused by other bacteria species (Table 3 ). The relative mortality risk within 1 year of revision was 3.10 (95\% CI, 1.66-5.81) times greater if the PJI was caused by enterococcus 
Table 2. Survival after primary THA and revision for PJI

\begin{tabular}{|c|c|c|c|}
\hline Time from primary THA & $\begin{array}{l}\text { Nonrevision population } \\
66,709 \text { THAs } \\
\text { Death rate }(\%) \\
(95 \% \mathrm{CI})\end{array}$ & $\begin{array}{l}\text { Aseptic revision population } \\
1350 \text { THAs } \\
\text { Death rate }(\%) \\
(95 \% \mathrm{CI})\end{array}$ & $\begin{array}{l}\text { Revision for PJI population } \\
445 \text { THAs } \\
\text { Death rate }(\%) \\
(95 \% \mathrm{CI})\end{array}$ \\
\hline 30 days & $0.65(0.59-0.71)$ & $0.81(0.45-1.47)$ & $1.11(0.47-2.68)$ \\
\hline 90 days & $1.28(1.20-1.37)$ & $2.22(1.56-3.16)$ & $3.37(2.05-5.53)$ \\
\hline 183 days & $1.85(1.75-1.96)$ & $3.48(2.63-4.61)$ & $5.84(4.02-8.46)$ \\
\hline 365 days & $2.86(2.74-2.99)$ & $4.89(3.86-6.18)$ & $7.64(5.52-10.5)$ \\
\hline
\end{tabular}

$\mathrm{PJI}=$ prosthetic joint infection.

Table 3. Mortality risk for different bacteria species causing PJI

\begin{tabular}{lllll}
\hline Bacteria & Number of revisions for PJI & Relative risk & 95\% CI & p Value \\
\hline Staphylococcus aureus & 127 & 1.00 & (reference) & $0.42-2.73$ \\
Coagulase negative Staphylococci & 83 & 1.07 & $0.26-4.45$ & 0.88 \\
Enterobacteriaceae & 24 & 1.07 & 0.92 \\
Enterococcus spp & 48 & 2.89 & $0.30-6.40$ & 0.01 \\
Streptococcus spp & 32 & 0.39 & $0.37-2.63$ & 0.37 \\
Polymicrobial & 76 & 0.98 & 0.97 & $0.32-4.94$ \\
Other & 22 & 1.26 & 0.32 \\
\hline
\end{tabular}

$\mathrm{PJI}=$ prosthetic joint infection.

Table 4. Type of preoperative prophylactic antibiotic in revisions for PJI

\begin{tabular}{lrr}
\hline Prophylactic antibiotic & Number of revisions & $\%$ \\
\hline Cefuroxime & 203 & 46 \\
Dicloxacillin & 123 & 28 \\
Cefuroxime + vancomycin & 37 & 8 \\
Vancomycin & 18 & 4 \\
Dicloxacillin + vancomycin & 16 & 4 \\
Cefuroxime + gentamicin & 11 & 2 \\
Other & 19 & 4 \\
None & 12 & 3 \\
Missing information & 6 & 1 \\
Total & 445 & 100 \\
\hline
\end{tabular}

compared with all other bacteria as a group ( $\mathrm{p}<0.001)$. For the patients who had revision for PJI infected with enterococci, 36 of the $48(75 \%)$ were treated exclusively with $\beta$-lactam antibiotics preoperatively (Table 4).

\section{Discussion}

Patients who undergo revision surgery for PJI have a higher risk of unplanned readmission [19] and longer length of stay [23] compared with patients who undergo revision surgery for other causes, which implies that PJI has a major effect on a patient's health. One study showed that revision for PJI in THAs or TKAs is associated with higher mortality than aseptic revision [27], but not all studies have found this association [4, 24]. None of the previous published studies exclusively investigated the associated between revision for early postoperative PJI in primary THA and mortality.

Our study showed that patients who acquire a PJI within 1 year after their primary THA have an increased mortality risk. The increased mortality risk is not related to the revision surgery exclusively, because patients with a revision performed for PJI have an increased mortality risk when compared with patients with aseptic revision surgery. Patients with an enterococci-infected THA were especially at risk for mortality.

This study has several limitations. The risk of misclassification of the outcome death is almost nonexistent [20], but the sensitivity of the validated PJI diagnosis is only $90 \%$ [7], which might cause some revisions for PJI to be misclassified as aseptic revisions. Thus, the incidence of PJI is probably underestimated and the relative mortality risk for revisions for PJI compared with aseptic revisions also might be underestimated. Conversely, regarding the relative mortality risk, the reverse effect also could be true; that the $10 \%$ misclassified PJIs may be misclassified because they are caused by low-virulence bacteria. These 
are associated with lower mortality than high-virulent bacteria causing us to overestimate the relative mortality risk associated with PJI. Nevertheless, this does not alter that patients with PJI have a higher mortality risk than patients without PJI as even patients undergoing revisions for PJI attributable to low-virulent bacteria most likely have a higher mortality risk than patients without PJI. The relative mortality risk for patients with PJI compared with the nonrevision population also might be underestimated because patients with nonsurgically treated PJIs were included in the nonrevision population; however, because the number of these most likely is limited [12] and probably has a different effect on the patients' health, we do not believe there is a great effect on our estimation. Some known important risk factors for PJI and mortality were not reported to the registers such as obesity, smoking, and alcohol abuse, which may result in unmeasured confounding. However, we did adjust for diabetes mellitus and chronic obstructive pulmonary disease, as these are included in the Charlson Comorbidity Index score. Although the indication for primary THA was known, we chose not to adjust for it as the number of events (deaths) in our study population limited the number of parameters for which we could adjust. This might have resulted in unmeasured confounding as, for example, femoral fractures are associated with an increased risk of PJI and death. This unmeasured confounding could result in an overestimation of the relative mortality risk. We did perform a second crude analysis including only patients with osteoarthritis but without adjusting for other confounders and found that it did not alter the result of a notability higher mortality associated with revision surgery for PJI. In this study we used cumulative competing risk analysis to estimate the PJI incidence, the Kaplan-Meier analysis to estimate the incidence of death, and the pseudovalue analysis to estimate the relative mortality risk. All three analyses are based on assumption of independent observations that are not entirely fulfilled for bilateral THAs. A previous study showed that the bilaterally issue in register settings has little practical consequence when the outcome studied is revision [17], but this might not be applicable to death, which affects both hips. A solution to this problem could be to include only one primary THA for each patient, but that would lead to selection bias. Of the 59,954 patients 8550 had bilateral primary THAs and of those 39 patients had a primary THA on both sides within 1 year and died within 1 year after the first primary THA. Therefore, we believe it is unlikely that including bilateral THAs had a strong influence on our results.

Patients with a revision surgery for PJI within 1 year of the primary THA had more than double relative mortality risk compared with the nonrevision population. This underlines that PJI is a devastating complication and should be a concern for patients and clinicians. Before undergoing a primary THA, patients with an increased risk of acquiring a PJI should be aware that not only is a PJI associated with increased risk of multiple revisions, readmissions to the hospital, and longer length-of-stay, it also is associated with an increased risk of death. The information also may be important for payment models depending on their construction. Treatment and research should focus on improving prevention and treatment of PJIs, for example, through more careful patient selection, identification of bacteria, and changes in practice management [10].

The increased relative mortality risk between revision for PJI and aseptic revisions we found is somewhat lower than the fivefold increase in mortality described by Zmistowski et al. [27]. The difference in study populations might explain the difference in estimates between their study and our study; we included only revisions performed within 1 year of THA, because these would be the most clinically relevant for physicians and patients who have to decide whether to perform a primary THA if known risk factors for PJI are present, and the mortality is more likely to be influenced by the infection and revision than later events. Moreover, in contrast to the study by Zmistowski et al. [27], who included TKAs and all revisions, only firsttime revisions and only THAs were included in our study. We structured our study in this manner because the risk of failure increases with each revision performed [5], and THAs and TKAs differ on several parameters, including the risk of PJI and length of stay after a revision [9]. Finally, in contrast to the study by Zmistowski et al. [27], Denmark has a rather homogeneous population and all Danish citizens have tax-supported free medical care.

Revisions for PJI caused by enterococci generally have a higher risk of failure $[13,18]$, and this study showed that PJI caused by enterococci also is associated with a higher risk of death. An explanation for the higher mortality could be that enterococci have intrinsic antimicrobial resistance to $\beta$-lactams, which were the most commonly used prophylactic antibiotics before revision surgery (Table 4). A $\beta$-lactam was used exclusively in $73 \%$ of the patients who had revision surgery (and in $75 \%$ of the patients who had revision surgery for PJI infected with enterococci). This might pose a problem in terms of antibiotic coverage in general because we also found a high percentage of coagulase-negative staphylococci, which has become increasingly resistant to $\beta$-lactams [14]. Adding vancomycin to the prophylactic treatment could be a solution [22], as was done in $16 \%$ of the patients who had revision surgery. However, patients treated with preoperative prophylactic vancomycin had a similar mortality risk compared with patients treated with other types of preoperative prophylactic antibiotics. Furthermore, using vancomycin as standard treatment will probably result in an increase of side effects and antimicrobial resistance [26]. A 
more-efficient strategy might be to increase the number of PJIs diagnosed before revision, (using arthrocentesis), to reduce the risk of disregarding a PJI as presumable aseptic loosening and increase the chance of identifying bacteria and antimicrobial resistance before surgery.

Charnley [3] reported that the greatest challenge of THA was to eliminate PJI completely, but the history of arthroplasty has shown that we most likely will never overcome this challenge, and PJI will always be a risk when performing THA. In this registry study, we found that revision for PJI within 1 year of a primary THA induces an approximately twofold increased mortality risk during the year after the revision procedure. We also found that PJI caused by enterococci was associated with increased mortality risk compared with PJI caused by other bacteria. This underlines the need for further studies on identification of prognostic markers (eg, bacteria with antimicrobial resistance to the administered preoperative prophylactic antibiotic) and at-risk patients associated with a high risk of mortality after PJI.

Acknowledgments We thank Henrik Carl Schønheyder MD, DMSc (Department of Clinical Microbiology, Aalborg University Hosptial, Denmark), Per Kjærsgaard-Andersen MD (Department of Orthopedics, Vejle Hospital, Denmark), and Jens Kjølseth Møller MD, DMSc (Department of Clinical Microbiology, Vejle Hospital, Denmark) for their contribution to the studies that rendered our study possible.

\section{References}

1. Andersen PK, Klein JP, Rosthøj S. Generalised linear models for correlated pseudo-observations, with applications to multi-state models. Biometrika. 2003;90:15-27.

2. Bank S, Soby KM, Kristensen LH, Voldstedlund M, Prag J. A validation of the Danish microbiology database (MiBa) and incidence rate of Actinotignum schaalii (Actinobaculum schaalii) bacteraemia in Denmark. Clin Microbiol Infect. 2015;21:1097.e1-4.

3. Charnley J. Postoperative infection after total hip replacement with special reference to air contamination in the operating room. Clin Orthop Relat Res. 1972;87:167-87.

4. Choi HR, Beecher B, Bedair H. Mortality after septic versus aseptic revision total hip arthroplasty: a matched-cohort study. $J$ Arthroplasty. 2013;28(8 suppl):56-58.

5. Danish Hip Arthroplasty Register: Annual Report 2016 Available at: https://www.sundhed.dk/content/cms/98/4698_dhr-\%C3\%A5 rsrapport-2016.pdf. 2016. Accessed August 1, 2016.

6. Gundtoft PH, Overgaard S, Schonheyder HC, Moller JK, Kjaersgaard-Andersen $\mathrm{P}$, Pedersen AB. The "true" incidence of surgically treated deep prosthetic joint infection after 32,896 primary total hip arthroplasties: a prospective cohort study. Acta Orthop. 2015;86:326-334.

7. Gundtoft PH, Pedersen AB, Schonheyder HC, Overgaard S. Validation of the diagnosis 'prosthetic joint infection' in the Danish Hip Arthroplasty Register. Bone Joint J. 2016;98:320-325.

8. Jamsen E, Huotari K, Huhtala H, Nevalainen J, Konttinen YT. Low rate of infected knee replacements in a nationwide series: is it an underestimate? Acta Orthop. 2009;80:205-212.
9. Kurtz SM, Lau E, Schmier J, Ong KL, Zhao K, Parvizi J. Infection burden for hip and knee arthroplasty in the United States. J Arthroplasty. 2008;23:984-991.

10. Lindberg-Larsen M, Jorgensen CC, Bagger J, Schroder HM, Kehlet H. Revision of infected knee arthroplasties in Denmark. Acta Orthop. 2016;87:333-338.

11. Lindgren JV, Gordon M, Wretenberg P, Karrholm J, Garellick G. Validation of reoperations due to infection in the Swedish Hip Arthroplasty Register. BMC Musculoskelet Disord. 2014;15:384.

12. Lindgren V, Gordon M, Wretenberg P, Karrholm J, Garellick G. Deep infection after total hip replacement: a method for national incidence surveillance. Infect Control Hosp Epidemiol. 2014;35:1491-1496.

13. Lora-Tamayo J, Euba G, Ribera A, Murillo O, Pedrero S, GarciaSomoza D, Pujol M, Cabo X, Ariza J. Infected hip hemiarthroplasties and total hip arthroplasties: Differential findings and prognosis. J Infect. 2013;67:536-544.

14. Lutro O, Langvatn H, Dale H, Schrama JC, Hallan G, Espehaug B, Sjursen H, Engesaeter LB. Increasing resistance of coagulasenegative staphylococci in total hip arthroplasty infections: 278 THA-revisions due to infection reported to the Norwegian Arthroplasty Register from 1993 to 2007. Adv Orthop. 2014;2014:580359.

15. Pedersen A, Johnsen S, Overgaard S, Soballe K, Sorensen HT, Lucht U. Registration in the danish hip arthroplasty registry: completeness of total hip arthroplasties and positive predictive value of registered diagnosis and postoperative complications. Acta Orthop Scand. 2004;75:434-441.

16. Quan H, Sundararajan V, Halfon P, Fong A, Burnand B, Luthi JC, Saunders LD, Beck CA, Feasby TE, Ghali WA. Coding algorithms for defining comorbidities in ICD-9-CM and ICD-10 administrative data. Med Care. 2005;43:1130-1139.

17. Ranstam J, Karrholm J, Pulkkinen P, Makela K, Espehaug B, Pedersen AB, Mehnert F, Furnes O. Statistical analysis of arthroplasty data: II. Guidelines. Acta Orthop. 2011;82:258-267.

18. Rasouli MR, Tripathi MS, Kenyon R, Wetters N, Della Valle CJ, Parvizi J. Low rate of infection control in enterococcal periprosthetic joint infections. Clin Orthop Relat Res. 2012;470: $2708-2716$.

19. Schairer WW, Sing DC, Vail TP, Bozic KJ. Causes and frequency of unplanned hospital readmission after total hip arthroplasty. Clin Orthop Relat Res. 2014;472:464-470.

20. Schmidt M, Pedersen L, Sorensen HT. The Danish Civil Registration System as a tool in epidemiology. Eur J Epidemiol. 2014;29:541-549.

21. Thygesen SK, Christiansen CF, Christensen S, Lash TL, Sorensen HT. The predictive value of ICD-10 diagnostic coding used to assess Charlson comorbidity index conditions in the populationbased Danish National Registry of Patients. BMC Med Res Methodol. 2011;11:83.

22. Trampuz A, Zimmerli W. Diagnosis and treatment of implantassociated septic arthritis and osteomyelitis. Curr Infect Dis Rep. 2008;10:394-403.

23. Vanhegan IS, Malik AK, Jayakumar P, Ul Islam S, Haddad FS. A financial analysis of revision hip arthroplasty: the economic burden in relation to the national tariff. J Bone Joint Surg Br. 2012;94:619-623.

24. Webb JE, Schleck CD, Larson DR, Lewallen DG, Trousdale RT. Mortality of elderly patients after two-stage reimplantation for total joint infection: a case-control study. $J$ Arthroplasty. 2014;29:2206-2210.

25. Zhou Z, Rahme E, Abrahamowicz M, Pilote L. Survival bias associated with time-to-treatment initiation in drug effectiveness evaluation: a comparison of methods. Am J Epidemiol. 2005;162:1016-1023. 
26. Zhu HX, Cai XZ. CORR Insights((R)): Addition of vancomycin to cefazolin prophylaxis is associated with acute kidney injury after primary joint arthroplasty. Clin Orthop Relat Res. 2015;473:2204-2206.
27. Zmistowski B, Karam JA, Durinka JB, Casper DS, Parvizi J. Periprosthetic joint infection increases the risk of one-year mortality. J Bone Joint Surg Am. 2013;95:2177-2184. 